ROME prep. $1200 / 98$

$\mathrm{SNS} / \mathrm{PH} / 1998-004$

\title{
On the Definition of Gauge Field Operators in Lattice Gauge-Fixed Theories
}

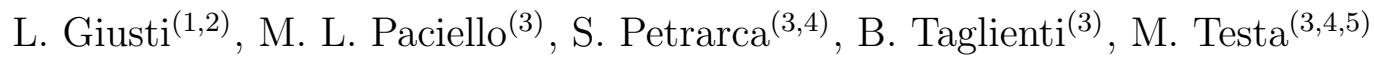 \\ ${ }^{1}$ Scuola Normale Superiore, P.zza dei Cavalieri 7, I-56100 Pisa Italy. \\ ${ }^{2}$ INFN, Sezione di Pisa, San Piero a Grado, I-56100 Pisa Italy. \\ ${ }^{3}$ INFN, Sezione di Roma 1, P.le A. Moro 2, I-00185 Roma, Italy. \\ ${ }^{4}$ Dipartimento di Fisica, Università di Roma "La Sapienza", \\ P.le A. Moro 2, I-00185 Roma, Italy. \\ 5 Theory Division, CERN, 1211 Geneva 23, Switzerland*.
}

\begin{abstract}
We address the problem of defining the four-potential, $A_{\mu}^{a}(x)$, on the lattice, in terms of the natural link variables, $U_{\mu}(x)$.

Different regularized definitions are shown, through non perturbative numerical computation, to converge towards the same continuum renormalized limit.
\end{abstract}

ROME prep. 1200/98

$\mathrm{SNS} / \mathrm{PH} / 1998-004$

^ Address until August 31st, 1998. 


\section{Introduction}

Lattice QCD does not require, in itself, any gauge-fixing in order to compute physical quantities. However, as became increasingly clear in recent years, lattice gaugefixing provides a necessary instrument in the study of quantities, like the quark and gluon propagators, whose behavior could be relevant to the study of the confinement mechanism [1]-[5].

Gauge-fixing is also a necessary ingredient in some non-perturbative renormalization schemes 6, 7] and it has been shown to facilitate the construction of composite fermion operators with correct chiral behavior[6], essential for the study of hadronic weak interaction phenomenology [8]-11].

Once gauge-fixing has been performed, Green's functions containing the fundamental quark and gluon field insertions, become accessible to non-perturbative study. However, contrary to what happens for quarks, a natural definition for the gluon field is missing. In the general field theoretical framework, as checked in perturbation theory, this is known not to be a real problem: any pair of operators differing from each other by irrelevant terms, i.e. formally equal up to terms of order $a$, will tend, to the same continuum operator, up to a constant. It is the purpose of this paper to show that this feature is also satisfied at the non-perturbative level, within the framework of lattice QCD. We will, in fact, show that different definitions of the gluon field, at the lattice level, give rise to Green's functions proportional to each other, thus guaranteeing the uniqueness of the renormalized continuum gluon field.

The plan of the paper is as follows. In section 2, we recall some basic facts about lattice gauge-fixing. In section 3 we discuss the ambiguities intrinsic to the lattice definition of gauge potential and in section 4 we summarize our results.

\section{Lattice Gauge-Fixing}

The Landau or Coulomb lattice gauge-fixing procedures are well known and several numerical algorithms are available to this aim. In the standard approach [1, 9] the functional:

$$
F\left[U^{\Omega}\right] \equiv-\frac{1}{V \cdot T} \operatorname{Re} \operatorname{Tr} \sum_{\mu} \sum_{x} U_{\mu}^{\Omega}(x)
$$

is minimized with respect to $\Omega(x)$. In eq.(11) $V$ is the lattice spatial volume, $T$ its time extension and $U_{\mu}^{\Omega}(x) \equiv \Omega(x) U_{\mu}(x) \Omega(x+\mu)^{\dagger}$ is the compact $S U(3)$ gauge field, gauge transformed by the local gauge transformation $\Omega(x)$. The extrema of $F$ with respect to $\Omega$, correspond to configurations satisfying the gauge condition $\partial_{\mu} A_{\mu}^{\Omega}=0$ in discretized form, with the lattice $A_{\mu}$ definition:

$$
A_{\mu}(x) \equiv \frac{\left(U_{\mu}(x)-U_{\mu}^{\dagger}(x)\right)_{\text {traceless }}}{2 i a g_{0}}, \quad \mu=1, \ldots 4,
$$

where $a$ is the lattice spacing and $g_{0}$ is the lattice bare coupling constant. The numerical behaviour of the gauge-fixing algorithm is usually monitored, as a function of the number of sweeps, by two quantities. The first is $F\left[U^{\Omega}\right]$ itself, and decreases 
monotonically. The second, denoted by $\theta$, is defined as:

$$
\theta \equiv \frac{1}{V \cdot T} \sum_{x} \theta(x) \equiv \frac{1}{V \cdot T} \sum_{x} \operatorname{Tr}\left[\Delta(x) \Delta^{\dagger}(x)\right]
$$

where:

$$
\Delta(x) \equiv \sum_{\mu}\left(A_{\mu}^{\Omega}(x)-A_{\mu}^{\Omega}(x-\hat{\mu})\right) .
$$

The functional $F\left[U^{\Omega}\right]$ is a lattice discretization of $\int d^{4} x \operatorname{Tr}\left(A_{\mu}^{2}\right)$, while $\theta$ corresponds to the continuum quantity $\int d^{4} x \operatorname{Tr}\left(\partial_{\mu} A_{\mu}\right)^{2}$. By its very definition, $\theta$, as a functional of $\Omega$, decreases (not strictly monotonically) during the gauge-fixing process, becoming zero when $F\left[U^{\Omega}\right]$ gets constant: its value controls the fulfillment of the gauge condition.

The main unsolved problems concerning gauge-fixing stem from the existence of both continuum and lattice Gribov copies 12]-15] and the numerical noise that they can generate.

Being a result of discretization effects, the lattice Gribov copies are of a quite different nature from those of the continuum, related to topological obstructions of the gauge-fixing condition $\partial_{\mu} A_{\mu}=0$ [13].

This confusing situation of the numerical gauge-fixing has been already stressed in the literature [16]. The real concern is, of course, the influence that these phenomena may have on the value of continuum observables, when computed through the intermediary of non gauge invariant quantities in the schemes referred to above.

\section{The Lattice Gauge Potential}

In this section we will discuss the problems related to the ambiguities in the lattice definition of the gauge potential.

A natural definition of the 4-potential in terms of the links, $U_{\mu}$, which represent the fundamental dynamical gluon variables, is given in eq.(2). This definition is naively suggested by the interpretation of $U_{\mu}(x)$ as the lattice parallel transport operator and by its formal expression in terms of the "continuum" gauge field variables, $A_{\mu}(x)$ as:

$$
U_{\mu}(x) \equiv \exp \left(i g_{0} a A_{\mu}(x)\right) \text {. }
$$

A formal expansion in powers of $a$, shows that eq.(2) defines $A_{\mu}(x)$, up to terms formally of order $a^{2}$.

It is clear that the definition given in eq.(2) is far from unique: it cannot be preferred to any other definition with analogue properties as, for instance:

$$
A_{\mu}^{\prime}(x) \equiv \frac{\left(\left(U_{\mu}(x)\right)^{2}-\left(U_{\mu}^{\dagger}(x)\right)^{2}\right)_{\text {traceless }}}{4 i a g_{0}}, \quad \mu=1, \ldots 4 \text {. }
$$

which in fact differs from the one given in eq.(2) by terms of $O(a)$ that formally go to zero as $a \rightarrow 0$.

¿From the algorithmical point of view, however, the various definitions are not interchangeable. In fact let us see what happens if we fix the gauge of a thermalized configuration stopping the gauge-fixing sweeps when $\theta \leq 10^{-14}$ and then define $\theta^{\prime}$ as having the same functional form of $\theta$, as in eq. (3), with $A_{\mu}$ replaced by $A_{\mu}^{\prime}$. 
The values of $\theta$ and $\theta^{\prime}$ during the minimization of $F$ are reported in Fig.1, for a typical thermalized configuration, as functions of the lattice sweeps of the numerical gauge-fixing algorithm. As clearly seen $\theta^{\prime}$ does not follow the same decreasing behaviour as $\theta$ : after an initial decrease, $\theta^{\prime}$ goes to a constant value, many orders of magnitude higher than the corresponding value of $\theta$. This fact has been already remarked in ref. [17] where it has been attributed to the large contribution of order $a$ terms.

This marked difference between the behavior of $\theta$ and $\theta^{\prime}$ seems to cast doubts on the lattice gauge-fixing procedure and on the corresponding continuum limit of gauge dependent operators. On the contrary, we will show, at the end of this section, that this discrepancy has a natural field theoretical explanation.

The relation between the two lattice definitions $A_{\mu}(x)$ and $A_{\mu}^{\prime}(x)$ is of the form:

$$
A^{\prime}{ }_{\mu}(x)=A_{\mu}(x)+a^{2} W_{\mu}(x)
$$

where the $W_{\mu}(x)$ is a dimension 3 operator $^{1}$ with the same quantum numbers of $A_{\mu}(x)$. The operator $W_{\mu}(x)$ in eq.(7) is not renormalized, so that, while formally it would seem safe to neglect its contribution in the continuum limit, in fact we must take it carefully into account. We start constructing, out of $W_{\mu}(x)$, a finite, renormalized operator, $W_{\mu}^{R}(x)$ as:

$$
W_{\mu}^{R}(x)=Z_{W}\left(g_{0}, a \mu_{R}\right)\left(W_{\mu}(x)+\frac{1-C\left(g_{0}\right)}{a^{2}} A_{\mu}(x)\right)
$$

where $\mu_{R}$ is the mass renormalization scale of the theory and $Z_{W}$ is a logarithmically divergent, subtraction dependent constant, while $C$, as a consequence of the CallanSymanzik equation, can only depend on the bare coupling $g_{0}$ [18]. Eqs.(77) and (8) show that

$$
A^{\prime}{ }_{\mu}(x)=C\left(g_{0}\right) A_{\mu}(x)+\frac{a^{2}}{Z_{W}} W_{\mu}^{R}(x)
$$

so that, up to terms truly of order $a^{2}$ :

$$
A^{\prime}{ }_{\mu}(x)=C\left(g_{0}\right) A_{\mu}(x)
$$

For Green's functions insertions we have, therefore, in general:

$$
\frac{\left\langle\ldots A_{\mu}^{\prime}(x) \ldots\right\rangle}{\left\langle\ldots A_{\mu}(x) \ldots\right\rangle}=C\left(g_{0}\right)
$$

We have numerically checked some consequences of eq.(11) by measuring on different lattices, whose characteristics are reported in Table 1, the following Green functions for $S U(3)$ in the Landau gauge with periodic boundary conditions:

$$
\begin{aligned}
\left\langle\mathcal{A}_{0} \mathcal{A}_{0}\right\rangle(t) & \equiv \frac{1}{V^{2}} \sum_{\mathbf{x}, \mathbf{y}} \operatorname{Tr}\left\langle A_{0}(\mathbf{x}, t) A_{0}(\mathbf{y}, 0)\right\rangle \\
\left\langle\mathcal{A}_{i} \mathcal{A}_{i}\right\rangle(t) & \equiv \frac{1}{3 V^{2}} \sum_{i} \sum_{\mathbf{x}, \mathbf{y}} \operatorname{Tr}\left\langle A_{i}(\mathbf{x}, t) A_{i}(\mathbf{y}, 0)\right\rangle
\end{aligned}
$$

\footnotetext{
${ }^{1}$ We are assuming that only one operator $W_{\mu}(x)$ is present, in order to simplify the presentation. In general several dimension 3 operators exist and their mixing has to be taken into account, but the conclusions remain unchanged.
} 


$$
\begin{aligned}
\langle\partial \mathcal{A} \partial \mathcal{A}\rangle(t) & \equiv \frac{1}{16 V^{2}} \sum_{\mu, \nu} \sum_{\mathbf{x}, \mathbf{y}} \operatorname{Tr}\left\langle\partial_{\mu} A_{\mu}(\mathbf{x}, t) \partial_{\nu} A_{\nu}(\mathbf{y}, 0)\right\rangle \\
\left\langle\mathcal{A}_{0} \partial \mathcal{A}\right\rangle(t) & \equiv \frac{1}{4 V^{2}} \sum_{\mu} \sum_{\mathbf{x}, \mathbf{y}} \operatorname{Tr}\left\langle A_{0}(\mathbf{x}, t) \partial_{\mu} A_{\mu}(\mathbf{y}, 0)\right\rangle \\
\left\langle\mathcal{A}_{i} \partial \mathcal{A}\right\rangle(t) & \equiv \frac{1}{12 V^{2}} \sum_{\mu, i} \sum_{\mathbf{x}, \mathbf{y}} \operatorname{Tr}\left\langle A_{i}(\mathbf{x}, t) \partial_{\mu} A_{\mu}(\mathbf{y}, 0)\right\rangle
\end{aligned}
$$

using both $A$ and $A^{\prime}$, as defined in eqs.(2) and (6). In eqs.(12)-(16) the trace is over the color indexes, $\mu$ and $\nu$ run from 1 to 4 and $i$ from 1 to 3 . Here and in the following we define $\mathcal{A}_{\mu}(t)=\sum_{\mathbf{x}} A_{\mu}(\mathbf{x}, t)$.

The correlators defined in eqs.(12)-(16), and in particular $\left\langle\mathcal{A}_{i} \mathcal{A}_{i}\right\rangle(t)$, are relevant to the investigation of the QCD gluon sector [1]-[5]. In this paper we will not be concerned with the interpretation of their $t$ behavior: our aim is to show their independence on the ambiguities related to the definition of the gluon field.

The proportionality factor, $C\left(g_{0}\right)$, may depend on the direction $\mu$, if the lattice breaks cubic symmetry. In our case, as shown in Table 1, two of the lattices have a time extension different from the spatial one, so that we have a coefficient $C_{0}\left(g_{0}\right)$ relating $A_{0}^{\prime}$ to $A_{0}$ and a different one, $C_{i}\left(g_{0}\right)$, connecting $A_{i}^{\prime}$ to $A_{i}$.

It is worth noting that $\left\langle\mathcal{A}_{0} \mathcal{A}_{0}\right\rangle(t)$, when evaluated through $A_{\mu}(x)$, should be constant in $t$ configuration by configuration, in virtue of the Landau gauge condition which, together with periodic boundary conditions, implies $\partial_{0} \mathcal{A}_{0}=0$. The same should be true, on average, when $A^{\prime}$ is used. The behaviour of these two correlators is shown in Fig. 2 for the run W60b where the errors have been evaluated through jacknife. Fig. 2 puts in evidence the flatness of $\left\langle\mathcal{A}_{0}^{\prime} \mathcal{A}_{0}^{\prime}\right\rangle$ and $\left\langle\mathcal{A}_{0} \mathcal{A}_{0}\right\rangle$. As remarked above, $\left\langle\mathcal{A}_{0} \mathcal{A}_{0}\right\rangle$ has to be constant configuration by configuration, which is verified with a precision of $\sim 10^{-6}$. More surprising is the fact that also $\left\langle\mathcal{A}_{0}^{\prime} \mathcal{A}_{0}^{\prime}\right\rangle$ turns out to be constant configuration by configuration at the level of $\sim 5 \%$, also because in this case the behavior of the control variable $\theta^{\prime}$, displayed in Fig. 1, shows that $A^{\prime}$ is far from satisfying the Lorentz condition on individual configurations.

For both correlators, the error is just due to fluctuation of their constant (in $t$ ) value, configuration by configuration.

In Table 1 we report the fit of the ratio:

$$
\frac{\left\langle\mathcal{A}_{i}^{\prime} \mathcal{A}_{i}^{\prime}\right\rangle}{\left\langle\mathcal{A}_{i} \mathcal{A}_{i}\right\rangle} \equiv C_{i}^{2}\left(g_{0}\right)
$$

as a constant in time. In Fig. 3 the Green function $\left\langle\mathcal{A}_{i}^{\prime} \mathcal{A}_{i}^{\prime}\right\rangle$ and the rescaled one $C_{i}^{2}\left(g_{0}\right)\left\langle\mathcal{A}_{i} \mathcal{A}_{i}\right\rangle$ are reported for the run W60b. The remarkable agreement between these two quantities confirms the proportionality shown in eq. (10).

As shown in Table 1, $C_{0}\left(g_{0}\right)$ and $C_{i}\left(g_{0}\right)$ coincide, within the errors, for the symmetric lattices W58 and W60a, while they have a different value for W60b and W64. This is probably due to the breaking of cubic symmetry. This interpretation is confirmed by considering the W60a $\left(8^{3} \cdot 8\right)$ and W60b $\left(8^{3} \cdot 16\right)$ lattices, with the same $\beta$ value and configured so that the time extension of W60a is equal to the spatial extension of W60b. In fact we find that the coefficients $C_{0}\left(g_{0}\right)$ evaluated from W60a and $C_{i}\left(g_{0}\right)$ estimated from W60b agree within the errors. 
The breaking of cubic symmetry, which depends on the ratio $T /(V)^{1 / 3}$, could be a potential source of systematic error in the non-perturbative evaluation of renormalization constants on asymmetric lattices.

The remaining Green functions, eqs.(14 16), which contain the insertion of $\partial_{\mu} A_{\mu}$ exhibit the expected behavior: the ones formed with $A_{\mu}$ fluctuate around zero at a level of $10^{-5}$, while those built through $A_{\mu}^{\prime}$ fluctuate around zero at a level of $10^{-2}-10^{-3}$.

We are now ready to show why the discrepancy between the values of $\theta$, relevant to control the gauge-fixing algorithm, and the expectation values of $\theta^{\prime}$, is natural. In fact we have:

$$
\begin{aligned}
\theta & =a^{4} \int d^{4} x\left(\partial_{\mu} A_{\mu}(x)\right)^{2} \\
\theta^{\prime} & =a^{4} \int d^{4} x\left(\partial_{\mu} A_{\mu}^{\prime}(x)\right)^{2}
\end{aligned}
$$

In other words, $\theta$ and $\theta^{\prime}$ are proportional to two different discretizations of the same continuum operator $\left(\partial_{\mu} A_{\mu}\right)^{2}$. However, while $\theta$ vanishes configuration by configuration, as a consequence of the gauge fixing procedure, $\theta^{\prime}$ is proportional to $\left(\partial_{\mu} A_{\mu}^{\prime}\right)^{2}$, which has the vacuum quantum numbers and mixes with the identity. The expectation value of $\left(\partial_{\mu} A_{\mu}^{\prime}\right)^{2}$, therefore, diverges as $\frac{1}{a^{4}}$ so that $\theta^{\prime}$ will stay finite, as $a \rightarrow 0$.

\section{Conclusions}

In this paper we investigated the problem of the definition of lattice operators converging, as $a \rightarrow 0$, to the fundamental continuum gauge fields. This construction is affected, at the regularized lattice level, by an enormous redundancy. However we found convincing non-perturbative evidence, based on numerical simulations, that this redundancy will be completely compensated, in the continuum, by the wave function renormalization needed in order to define finite Green's functions with gauge field insertions. Although, on general field theoretical grounds, the validity of such results is not unexpected, we stress the remarkable fact that it holds true also in this particular situation in which gauge-fixing is naively performed, disregarding the problems related to the existence of lattice and continuum Gribov copies.

We have also discussed and solved the problem of the large discrepancy between the values of the control functionals $\theta$ and $\theta^{\prime}$ in terms of renormalization of power-divergent composite operators.

A direction dependent effect in the value of the renormalization constants has been found on non-cubic lattices, which should caution against too naive application of infinite volume results to finite volume lattice numerical data.

We believe that these features have a general validity and will survive a more thorough treatment of the gauge-fixing problem.

\section{Acknowledgements}

Massimo Testa thanks the CERN Theory Division for the kind hospitality. 


\section{References}

[1] J. E. Mandula, M. Ogilvie, Phys. Lett. B185 (1987) 127.

[2] R. Gupta, G. Guralnik, G. Kilcup, A. Patel, S. R. Sharpe, T. Warnock, Phys. Rev. D36 (1987) 2813.

[3] P. Marenzoni, G. Martinelli, N. Stella, M. Testa, Phys. Lett. B318 (1993) 511;

P. Marenzoni, G. Martinelli, N. Stella, Nucl. Phys. B455 (1995) 339.

[4] C. Bernard, C. Parrinello, A. Soni, Nucl. Phys. B (Proc. Suppl.) 30 (1993) 535; Phys. Rev. D49 (1994) 1585

[5] C. Bernard, A. Soni and K. Yee, Nucl. Phys. B (Proc. Suppl.) 20 (1991) 410.

[6] G. Martinelli, C. Pittori, C. T. Sachrajda, M. Testa, A. Vladikas, Nucl. Phys. B445 (1995) 81.

[7] B. Alles, D. S. Henty, H. Panagopoulos, C. Parrinello, C. Pittori, D. G. Richards: $\alpha_{s}$ from the Non-perturbatively Renormalised Lattice Three-gluon Vertex, heplat/9605033.

[8] M. Ciuchini, E. Franco, G. Martinelli, L. Reina, L. Silvestrini, Z. Phys. C68 (1995) 239.

[9] C. T. H. Davies, G. G. Batrouni, G. R. Katz, A. S. Kronfeld, G. P. Lepage, K. G. Wilson, P. Rossi, B. Svetitsky, Phys. Rev. D37 (1988) 1581.

[10] V. Giménez, L. Giusti, F. Rapuano, M. Talevi, Lattice Quark Masses: a Nonperturbative Measurement, hep-lat/9801028.

[11] L. Conti, A. Donini, V. Giménez, G. Martinelli, M. Talevi, A. Vladikas, Lattice B-parameters for $\Delta S=2$ and $\Delta I=\frac{3}{2}$ Operators, hep-lat/9711053.

[12] V. N. Gribov, Nucl. Phys. B139 (1978) 1;

For a review of the Gribov ambiguity in the continuum see for example S. Sciuto, Phys. Rep. 49 (1979) 181 and references therein.

[13] P. van Baal in NATO Avanced Study Institute on Confinement, Duality and Nonperturbative Aspects of QCD, Cambridge, England, 23 Jun - 4 Jul 1997 ,hepth/9711070.

[14] E. Marinari, C. Parrinello, R. Ricci, Nucl. Phys. B362 (1991) 487.

[15] C. Parrinello, S. Petrarca, A. Vladikas, Phys. Lett. B268 (1991) 236.

[16] See for example: J. E. Hetrick, Ph. de Forcrand, Smeared Gauge-Fixing, Talk given at Lattice 97: 15th International Symposium on Lattice Field Theory, Edinburgh, Scotland, 22-26 Jul 1997, hep-lat/9710003; W. Bock, M. Golterman, Y. Shamir, Gauge Fixing Approach to Lattice Chiral Gauge Theories, Talk given at Lattice 97: 15th International Symposium on Lattice Field Theory, Edinburgh, Scotland, 22-26 Jul 1997, hep-lat/9709113.

[17] L. Giusti, Nucl. Phys. B498 (1997) 331.

[18] M. Testa, Some Observations on Broken Symmetries, hep-th/9803147.

[19] K. D. Born, R. Altmeyer, W. Ibes, E. Laermann, R. Sommer, T.F. Walsh, P.M. Zerwas, Nucl. Phys. B (Proc. Suppl.) 20 (1991) 394.

[20] G. Bali, K. Schilling, Phys. Rev. D47 (1993) 661. 


\begin{tabular}{||c|cccc||}
\hline \hline & W58 & W60a & W60b & W64 \\
\hline$\beta$ & 5.8 & 6.0 & 6.0 & 6.4 \\
$\#$ Confs & 20 & 100 & 50 & 30 \\
Volume & $6^{3} \times 6$ & $8^{3} \times 8$ & $8^{3} \times 16$ & $8^{3} \times 16$ \\
\hline$C_{i}\left(g_{0}\right)$ & $0.689(3)$ & $0.729(1)$ & $0.729(2)$ & $0.757(2)$ \\
$C_{0}\left(g_{0}\right)$ & $0.690(7)$ & $0.729(1)$ & $0.750(1)$ & $0.784(2)$ \\
\hline$a^{-1}$ & $1.333(6)$ & $1.94(5)$ & $1.94(5)$ & $3.62(4)$ \\
\hline
\end{tabular}

Table 1: Summary of the lattice parameters used and relative values of $C_{0}$ and $C_{i}$ obtained as discussed in section 3. The $a^{-1}$ values are taken from [19, 20] and are computed through the string tension.

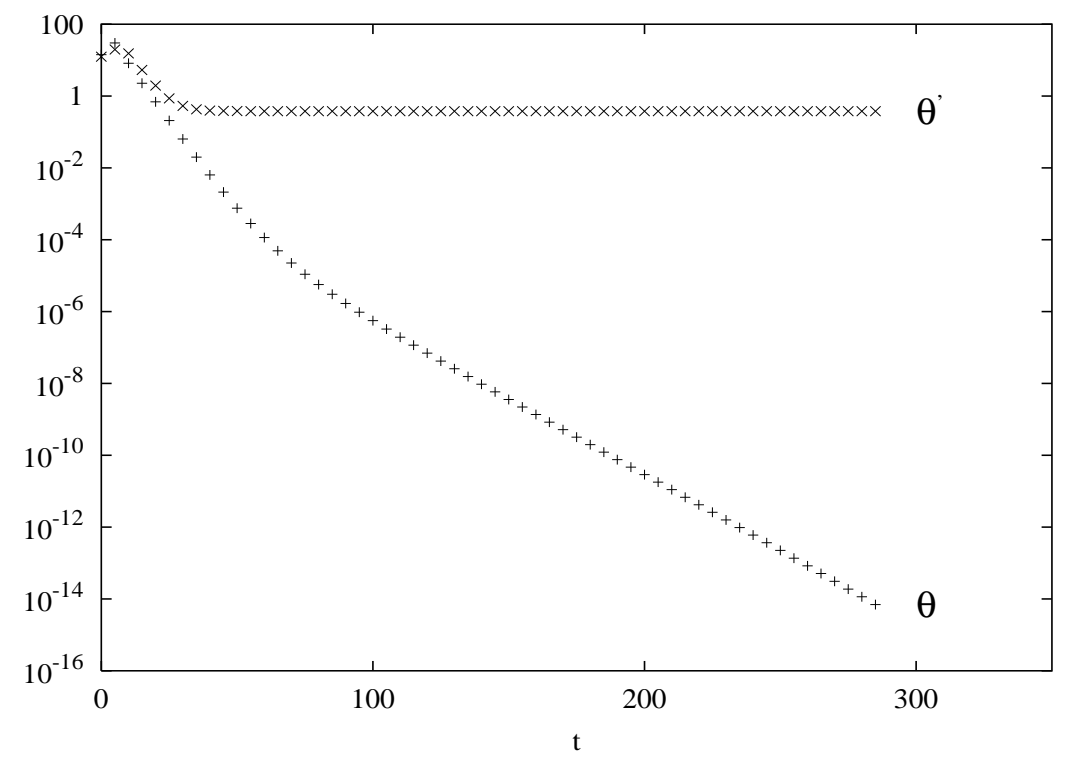

Figure 1: Typical behaviour of $\theta$ and $\theta^{\prime}$ vs gauge fixing sweeps at $\beta=6.0$ for a thermalized $S U(3)$ configuration $8^{3} \cdot 16$. 


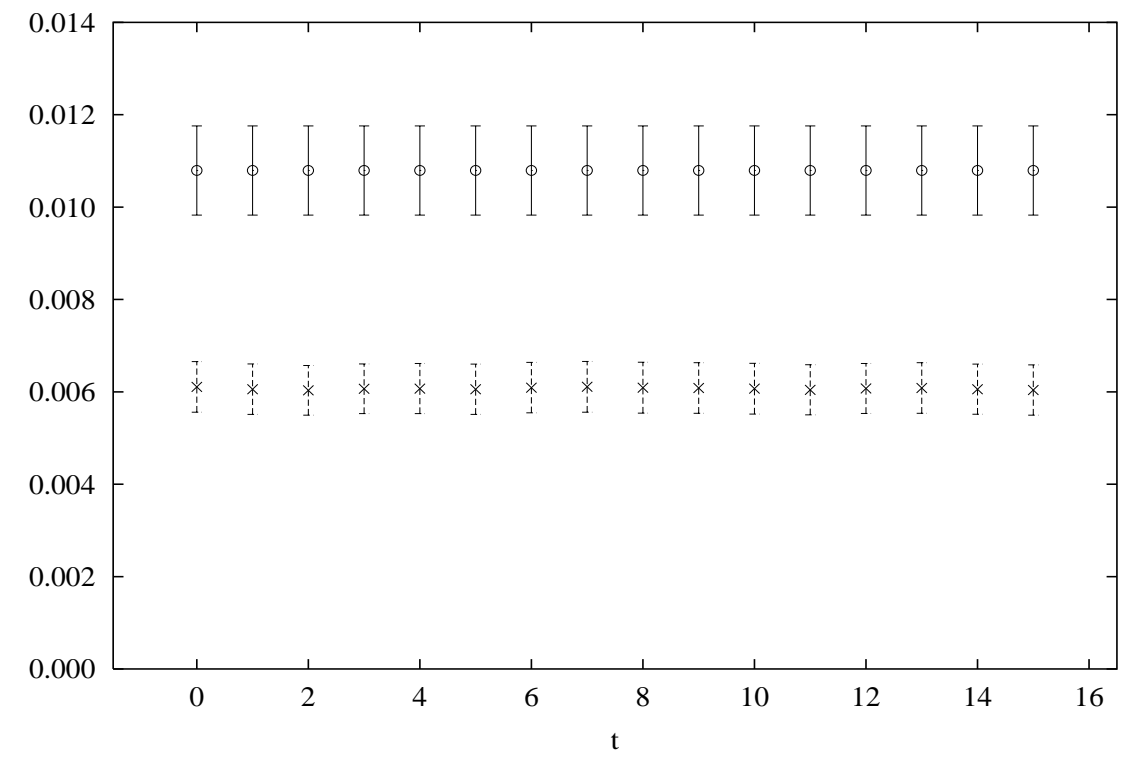

Figure 2: Comparison of the matrix elements of $\left\langle\mathcal{A}_{0} \mathcal{A}_{0}\right\rangle(t)$ (open circles) and $\left\langle\mathcal{A}_{0}^{\prime} \mathcal{A}_{0}^{\prime}\right\rangle(t)$ (crosses) as function of time for a set of 50 thermalized $S U(3)$ configurations at $\beta=6.0$ with a volume $V \cdot T=8^{3} \cdot 16$ (run $\left.W 60 b\right)$; the errors are jacknife.

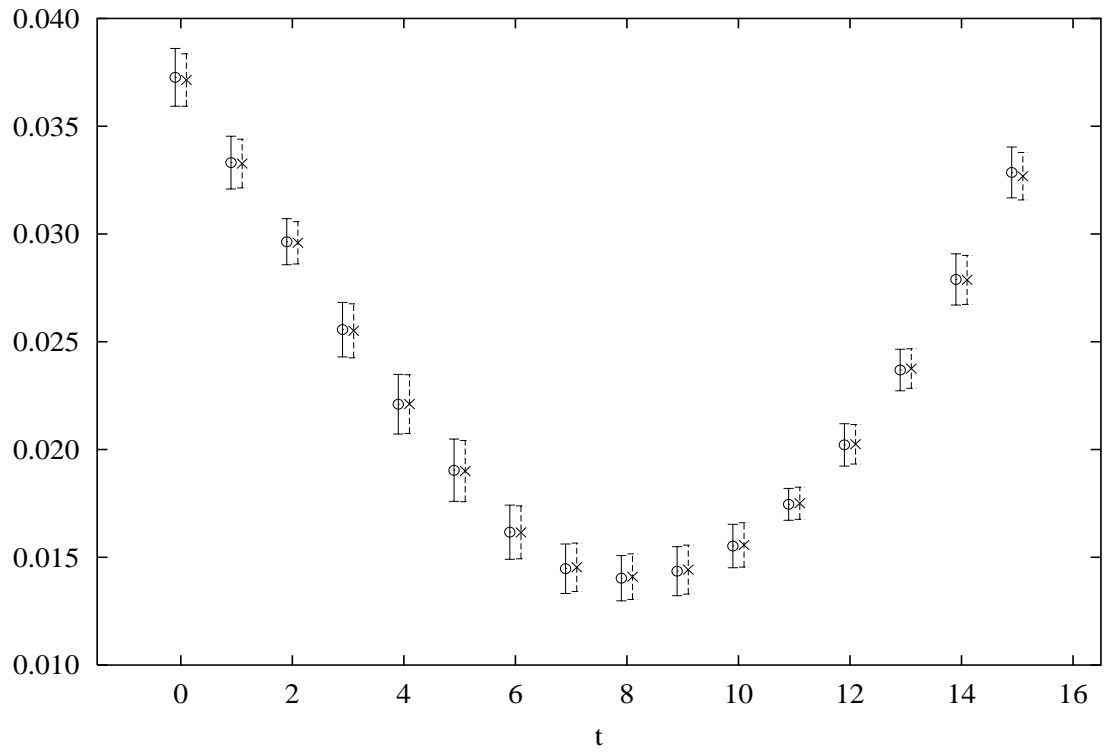

Figure 3: Comparison of the matrix elements of $\left\langle\mathcal{A}_{i}^{\prime} \mathcal{A}_{i}^{\prime}\right\rangle(t)$ (crosses) and the rescaled $\left\langle\mathcal{A}_{i} \mathcal{A}_{i}\right\rangle \cdot C_{i}^{2}\left(g_{0}\right)$ (open circles) as function of time for a set of 50 thermalized $S U(3)$ configurations at $\beta=6.0$ with a volume $V \cdot T=8^{3} \cdot 16$ (run $W 60 b$ ). The data have been slightly displaced in $t$ to help eye, the errors are jacknife. 